CITUSC/00-012

USC-00/01

hep-th/0002192

\title{
A New Supersymmetric Compactification of Chiral IIB Supergravity
}

\author{
Krzysztof Pilch and Nicholas P. Warner \\ Department of Physics and Astronomy \\ and \\ CIT-USC Center for Theoretical Physics \\ University of Southern California \\ Los Angeles, CA 90089-0484, USA
}

\begin{abstract}
We present a new compactification of chiral, $\mathcal{N}=2$ ten-dimensional supergravity down to five dimensions and show that it corresponds to the $\mathcal{N}=2$ supersymmetric critical point of five-dimensional, $\mathcal{N}=8$ gauged supergravity found in [1]. This solution presented here is of particular significance because it involves non-zero tensor gauge fields and, via the AdS/CFT correspondence, is dual to the non-trivial $\mathcal{N}=1$ supersymmetric fixed point of $\mathcal{N}=4$ Yang-Mills theory.
\end{abstract}

February, 2000 


\section{Introduction}

The generalizations of the AdS/CFT correspondence (see, e.g., [2] and the references therein) to theories with a scale has proven a rather successful enterprise over the last year. In particular, much work has been done on using supergravity to describe renormalization group flows of large $N$ field theories. One of the basic approaches to this has been to take a conformal field theory for which the correspondence has been well established and then perturb it by one or more relevant operators and use supergravity to follow the flow to the infra-red. The first supersymmetric flow to be described in terms of supergravity was discussed in [3]: The starting point of the flow was $\mathcal{N}=4$ Yang-Mills theory, while the end-point was an $\mathcal{N}=1$ superconformal fixed point identified in field theory in [4,5.6, 3].

Five-dimensional, $\mathcal{N}=8$ gauged supergravity is an invaluable tool in studying flows of the $\mathcal{N}=4$ Yang-Mills theory. The former field theory arises in the $S^{5}$ compactification of IIB supergravity as the field theory of the five-dimensional, $\mathcal{N}=8$ graviton supermultiplet. This is dual to the energy-momentum tensor supermultiplet of $\mathcal{N}=4$ Yang-Mills, and in particular the supergravity scalars are dual to gauge invariant, bilinear operators of the Yang-Mills theory. Thus supergravity scalars can be used to describe mass terms and vevs as well as the Yang-Mills coupling. The important point is that the five-dimensional, $\mathcal{N}=8$ gauged supergravity is, almost certainly a consistent truncation of the ten-dimensional, IIB theory, and therefore the equations of motion of the five-dimensional theory are embedded in those of the ten-dimensional theory to the extent that a solution of the former implies a solution of the latter. This means that the complexities of the ten-dimensional theory (on this special subsector) can be simplified by working entirely with the five-dimensional theory.

This was the approach taken in [1], where new critical points of the five-dimensional scalar potential were given. According to consistent truncation, these critical points must correspond to solutions of the IIB theory, and indeed this has been shown for the simpler critical points [7]:8]. From the AdS/CFT correspondence one should expect these critical points to correspond to new phases of the Yang-Mills theory. There is, however, one caveat: vacua that are unstable in supergravity exhibit apparently non-unitary behaviour in the corresponding Yang-Mills phases. At present the meaning of critical points is less than clear, but as vacua they are pathological, and so should be discarded. On the other hand, supersymmetric vacua in supergravity are known to be completely semi-classically stable [9]

[. It was argued in [1, 10] that supersymmetric critical points should always represent new

1 By an interesting "coincidence" (?) the only known perturbatively stable critical points are the supersymmetric ones. 
phases of the Yang-Mills theory even at finite $N$. This is because supersymmetric ground states of IIB supergravity should provide ground states for the IIB string, and hence the Yang-Mills phase should survive at finite N. By extrapolation, the non-supersymmetric, unstable critical points cannot be good string vacua, and so are most likely an example of "large N pathology."

It was for the foregoing reasons that the new $\mathcal{N}=2$ supersymmetric critical point in [1] was particularly significant. Moreover the subsequent analysis of that critical point, and the flow to it in supergravity matched perfectly with corresponding results in field theory providing a highly non-trivial test of the extension of the AdS/CFT correspondence to theories with a scale.

Our purpose in this paper is to return to the supersymmetric critical point in [1] and construct the exact corresponding ten-dimensional solution. In five dimensions this solution corresponded to turning on two scalar fields, but in ten-dimensions the background is rather more complex. The metric is deformed from the round $S^{5}$, and there are warp

factors, but the really new feature is that the non-zero fermion masses in the Yang-Mills theory mean that there must be non-zero $B_{\mu \nu}$ fields in ten-dimensions. There are few, if any, such backgrounds known that are also supersymmetric. Thus it is intrinsically interesting to exhibit such a ten-dimensional solution. Moreover, it is even more significant in that this solution represents the supergravity (and string theory) dual of the LeighStrassler fixed point. Preliminary work towards finding the ten-dimensional solution was done in [11], where the metric was computed using the formula given in [1], and a linearized form for the $B$-field was suggested.

Our analysis will proceed as follows: In section 2 we first obtain the ten-dimensional metric using the result in [1], and we give the Ricci tensor for this metric. Then, in section 3 we use the symmetries of the five-dimensional solution to arrive at an Ansatz for the tensor gauge fields. We then solve the ten-dimensional equations and exhibit the solution. In section 4 we confirm that our solution does indeed have the proper amount of supersymmetry, and give explicit formulae for the generators.

\section{The scalars and the metric}

We first, briefly review the structure of the five-dimensional solution, paying particular attention to its symmetries as they will be important later. 


\subsection{The five-dimensional solution and its symmetries}

In terms of Yang-Mills theory, the solution involves giving a mass to s single $\mathcal{N}=1$ hypermultiplet. That is, one perturbs the Hamiltonian by the fermion bilinear $\operatorname{Tr}\left(\lambda^{4} \lambda^{4}\right)$, and by the scalar field counterpart: $\operatorname{Tr}\left(X^{5} X^{5}+X^{6} X^{6}\right)$. We will denote the corresponding supergravity fields by $\chi$ and $\alpha$, respectively. 2 The first of these fields breaks the $S O(6)$ $R$-symmetry to $S U(3)$, while the second field breaks the $S O(6)$ down to $S O(4) \times S O(2)$. The two together break the $S O(6)$ to the group $S U(2) \times U(1)$, where the $U(1)$ is the $R$-symmetry at the new $\mathcal{N}=1$ Yang-Mills fixed point.

To see this more explicitly, think of $S O(6)$ as $S U(4)$ on the fundamental, then the $S U(3)$ is the upper-left $3 \times 3$ block, while $S O(4) \times S O(2) \equiv S U(2) \times S U(2) \times U(1)$ acts as two $2 \times 2$ blocks, with the $U(1)$ as $\operatorname{diag}\left(e^{i \phi}, e^{i \phi}, e^{-i \phi}, e^{-i \phi}\right)$. The $S U(2) \times U(1)$ invariance is the common subgroup consisting of the upper-left $S U(2)$ and the $U(1): \operatorname{diag}\left(e^{i \phi}, e^{i \phi}, e^{-2 i \phi}, 1\right)$.

If one takes into account the $S L(2, \mathbb{R})$ symmetry of the supergravity then there is a further $U(1)$ symmetry. The supergravity scalar $\alpha$ is $S L(2, \mathbb{R})$ invariant, but $\chi$ is part of an $S L(2, \mathbb{R})$ doublet, and may be taken to have charge +1 under $U(1) \subset S L(2, \mathbb{R})$. This can be cancelled by the action of the $U(1)$ factor in $S U(3) \times U(1) \subset S U(4)$.

On this two parameter subspace the supergravity potential takes the form [1]:

$$
V=-\frac{g^{2}}{4 \rho^{4}}\left(1-\sinh ^{4}(\chi)\right)-\frac{g^{2}}{2} \rho^{2} \cosh ^{2}(\chi)+\frac{g^{2}}{32} \rho^{8} \sinh ^{2}(2 \chi),
$$

and the superpotential is:

$$
W=\frac{1}{4 \rho^{2}}\left[\cosh (2 \chi)\left(\rho^{6}-2\right)-\left(3 \rho^{6}+2\right)\right] .
$$

where $\rho=e^{\alpha}$. The potential and superpotential are related via:

$$
V=\frac{g^{2}}{8}\left|\frac{\partial W}{\partial \chi}\right|^{2}+\frac{g^{2}}{48}\left|\frac{\partial W}{\partial \alpha}\right|^{2}-\frac{g^{2}}{3}|W|^{2} .
$$

The $\mathcal{N}=2$ supersymmetric critical point occurs at:

$$
\chi=\log (3) / 2, \quad \alpha=\log (2) / 6 .
$$

2 In [3] these were denoted by $\varphi_{1}$ and $\alpha$. 
Let $\Lambda$ and $\Lambda_{0}$ be the cosmological constants at the new critical point, and at the maximally supersymmetric point $(\alpha=\chi=0)$ respectively, then:

$$
\frac{\Lambda}{\Lambda_{0}}=\frac{V}{V_{0}}=\frac{42^{4 / 3}}{9}
$$

\subsection{The metric in ten-dimensions}

The ten-dimensional space-time we seek is a "warped" product $A d S_{5} \times M_{5}$ with the metric of the form:3

$$
d s_{10}^{2}=\Omega^{2} d s_{\mathrm{AdS}}^{2}-d s_{5}^{2}
$$

where $d s_{\text {AdS }}^{2}$ is the metric on $A d S_{5}, d s_{5}^{2}$ is the metric on the internal manifold, $M_{5}$, which is topologically a sphere.

The function, $\Omega$, is the warp factor and it depends on the coordinates of the internal manifold. Following [3], we take a coordinate system in which the AdS metric takes the form:

$$
d s_{\mathrm{AdS}}^{2}=e^{2 A(r)}\left(d x_{\mu} d x^{\mu}\right)-d r^{2}, \quad A(r)=\frac{r}{L}
$$

Consistent truncation directly determines the internal metric of (2.6) in terms of the scalar fields. The formula was obtained in [1], and its derivation is exactly parallel to the corresponding four-dimensional result [12]. The formula expresses the deformed metric in terms of bilinears in the Killing vectors, and thus it is simplest to start by taking coordinates in which these Killing vectors are as simple as possible. The natural choice is to think of $S^{5}$ as the surface $\sum_{I=1}^{6}\left(x^{I}\right)^{2}=1$ in $\mathbb{R}^{6}$, and use the cartesian coordinates. We obtain the following metric:

$$
d s_{5}^{2}(\alpha, \chi)=\frac{a^{2}}{2} \frac{\operatorname{sech} \chi}{\xi}\left(d x^{I} Q_{I J}^{-1} d x^{J}\right)+\frac{a^{2}}{2} \frac{\sinh \chi \tanh \chi}{\xi^{3}}\left(x^{I} J_{I J} d x^{J}\right)^{2}
$$

where $Q$ is a diagonal matrix with $Q_{11}=\ldots=Q_{44}=e^{-2 \alpha}$ and $Q_{55}=Q_{66}=e^{4 \alpha}, J$ is an antisymmetric matrix with $J_{14}=J_{23}=J_{65}=1, \xi^{2}=x^{I} Q_{I J} x^{J}$. The warp factor is simply

$$
\Omega^{2}=\xi \cosh \chi
$$

3 We use the $(+,-, \ldots,-)$ convention for the metric. 
The constant, $a$, has been introduced in (2.8) to account for the arbitrary normalization of the Killing vectors. The constant can be fixed by ensuring that (2.6) and (2.8) yield the proper metric for the round $S^{5}$ compactification $(\alpha=\chi=0)$. Specifically, one finds:

$$
a=\sqrt{2} L_{0}
$$

where $L_{0}$ is the radius of $A d S_{5}$ at the maximally supersymmetric point.

The metric (2.8) is valid for any configuration of scalar fields, $\alpha$ and $\chi$, including ones that depend on space-time coordinates. It is invariant under $S U(2) \times U(1) \times U(1)$ : The tensor $Q$ is manifestly $S U(2) \times S U(2) \times U(1)$ invariant, while $J$ is invariant under $S U(3) \times U(1)$, with the last $U(1)$ generated by $J$ itself.

We now pass to coordinates that are adapted to this isometry. Introduce the complex coordinates based on the complex structure $J$ :

$$
u^{1}=x^{1}+i x^{4}, \quad u^{2}=x^{2}+i x^{3}, \quad u^{3}=x^{5}-i x^{6}
$$

Now use the group action to reparamerize these coordinates, i.e. let:

$$
\left(\begin{array}{l}
u^{1} \\
u^{2}
\end{array}\right)=e^{-i \phi / 2} \cos \theta g\left(\alpha_{1}, \alpha_{2}, \alpha_{3}\right)\left(\begin{array}{l}
1 \\
0
\end{array}\right), \quad u^{3}=e^{-i \phi} \sin \theta
$$

where $g\left(\alpha_{1}, \alpha_{2}, \alpha_{3}\right)$ is an $S U(2)$ matrix expressed in terms of your Euler angles.

Performing the change of variables and restricting to the critical point we obtain

$$
\begin{aligned}
d s_{5}^{2}= & \frac{\sqrt{3}}{8} a^{2}(3-\cos (2 \theta))^{1 / 2}\left(d \theta^{2}+\frac{\cos ^{2} \theta}{3-\cos (2 \theta)}\left(\left(\sigma^{1}\right)^{2}+\left(\sigma^{2}\right)^{2}\right)+\frac{\sin ^{2}(2 \theta)}{(3-\cos (2 \theta))^{2}}\left(\sigma^{3}\right)^{2}\right) \\
& +\frac{\sqrt{3}}{12} a^{2}(3-\cos (2 \theta))^{1 / 2}\left(d \phi+\frac{2 \cos ^{2} \theta}{3-\cos (2 \theta)} \sigma^{3}\right)^{2},
\end{aligned}
$$

where $\sigma^{i}, i=1,2,3$, are the $S U(2)$ invariant forms satisfying $d \sigma^{i}=\sigma^{j} \wedge \sigma^{k}$, and

$$
\Omega^{2}=2^{1 / 3}\left(1-\frac{1}{3} \cos (2 \theta)\right)^{1 / 2}
$$

The $S U(2)$ invariance is manifest, and the two $U(1)$ 's correspond to a $\phi$-translation and the rotation of $\sigma^{1}$ into $\sigma^{2}$. For future reference we will call these rotations $U_{\phi}(1)$ and $U_{\sigma}(1)$ respectively. 
We choose orthogonal frames $e^{M}=\left(e^{m}, e^{a}\right), m=1, \ldots, 5, a=6, \ldots, 10$, for the metric (2.6) such that

$$
\begin{aligned}
& e^{1} \propto d x^{0}, \quad e^{2} \propto d x^{1}, \quad e^{3} \propto d x^{2}, \quad e^{4} \propto d x^{3}, \quad e^{5} \propto d r, \\
& e^{6} \propto d \theta, \quad e^{7} \propto \sigma^{1}, \quad e^{8} \propto \sigma^{2}, \quad e^{9} \propto \sigma^{3}, \quad e^{10} \propto d \phi+\ldots .
\end{aligned}
$$

An explicit evaluation of the Ricci tensor yields the following nonvanishing components

$$
\begin{gathered}
R_{11}=-R_{22}=\ldots=-R_{55} \\
=\frac{4}{L^{2}} \Omega(\theta)^{-2}-\frac{4}{\tilde{a}^{2}} \Omega(\theta)^{-10}(\cos (4 \theta)-10 \cos (2 \theta)+5), \\
R_{66}=R_{99}=-\frac{8}{3 \tilde{a}^{2}} \Omega(\theta)^{-10}(9 \cos (2 \theta)-31), \\
R_{77}=R_{88}=\frac{4}{3 \tilde{a}^{2}} \Omega(\theta)^{-10}(\cos (4 \theta)-18 \cos (2 \theta)+61), \\
R_{1010}=\frac{4}{3 \tilde{a}^{2}} \Omega(\theta)^{-10}(5 \cos (4 \theta)-54 \cos (2 \theta)+117),
\end{gathered}
$$

and

$$
R_{910}=\frac{4}{\tilde{a}^{2}} \sqrt{\frac{2}{3}} \Omega(\theta)^{-10}(\sin (4 \theta)-6 \sin (2 \theta)),
$$

where

$$
\tilde{a}^{2}=\frac{27}{42^{2 / 3}} a^{2}
$$

\section{The Ansatz and the solution}

The bosonic field equations of the IIB supergravity, with the dilaton and axion set to zero read [13:

$$
\begin{gathered}
R_{M N}=\frac{1}{6} F_{M Q R S}^{P F_{P Q R S N}}+\frac{1}{8}\left(G_{M Q}^{P Q} G_{P Q N}^{*}+G_{M}^{* P Q}{ }_{M} G_{P Q N}-\frac{1}{6} g_{M N} G^{P Q R} G_{P Q R}^{*}\right) \\
F_{M N P Q R}=\frac{1}{5 !} e \epsilon_{M N P Q R S T U V W} F^{S T U V W} \\
\nabla^{P} G_{M N P}=-\frac{2}{3} i F_{M N P Q R} G^{P Q R}
\end{gathered}
$$

and

$$
G^{P Q R} G_{P Q R}=0,
$$


together with Bianchi identities

$$
d F=\frac{1}{8} \operatorname{Im} G \wedge G^{*}
$$

and

$$
d G=0 .
$$

We are seeking a solution in which the 5-form field, $F$, is invariant under the symmetries of $A d S_{5}$. This leads to a generalized Freund-Rubin Ansatz [14,7] that is consistent with the warped product form of the metric and satisfies the self-dual field equations:

$$
F=-m \Omega^{-5}\left(e^{1} \wedge \ldots \wedge e^{5}+e^{6} \wedge \ldots \wedge e^{10}\right)
$$

To make the Ansatz for the potential, $B$, of the 3-index tensor, $G$, one starts by considering $G$ at the linearized level. The relevant harmonics lie in the $\mathbf{1 0}$ and $\overline{\mathbf{1 0}}$ of $S O(6)$, and in the cartesian coordinates these can be represented as self-dual and antiself-dual combinations of $d x^{I} \wedge d x^{J} \wedge d x^{K}$. We want the $S U(3)$ invariant combination $d u^{1} \wedge d u^{2} \wedge d u^{3}$ (and its complex conjugate). Writing this in terms of the coordinates (2.12) one finds the natural combination of the left invariant 1-forms: $\sigma^{1}-i \sigma^{2}$, which transforms with charge -1 under $U_{\sigma}(1)$. We also find that $G_{\text {linear }}=d u^{1} \wedge d u^{2} \wedge d u^{3}$ depends upon $\phi$ as an overall phase of $e^{-2 i \phi}$. The $U(1)$ invariance of $G_{\text {linear }}$ is thus a combination the $U_{\sigma}(1)$ with parameter $\beta$ and $U_{\phi}(1)$ with parameter $-\beta / 2$. This must also be an invariance of the full $G$ tensor.

Recall now that in the IIB theory, the $G$ and $G^{*}$ tensors form an $S L(2, \mathbb{R})$ doublet, with $G$ having charge +1 under $U(1) \in S L(2, \mathbb{R})$. Using this $U(1)$ we must be able to promote $U_{\sigma}(1)$ and $U_{\phi}(1)$ to full invariances of $G$. Put another way the complete $G$ tensor must transform with the same phases as $G_{\text {linear }}$ under $U_{\sigma}(1)$ and $U_{\phi}(1)$. This means that the complete $G$ must depend upon $\phi$ as an overall phase $e^{-2 i \phi}$, and must involve $\sigma^{1}$ and $\sigma^{2}$ only in the combination: $\sigma^{1}-i \sigma^{2}$.

This leads to the following Ansatz for the potential, $B$, of the 3-index tensor:

$$
B=e^{-2 i \phi}\left(a_{1}(\theta) d \theta \wedge\left(\sigma^{1}-i \sigma^{2}\right)+a_{2}(\theta)\left(\sigma^{1}-i \sigma^{2}\right) \wedge \sigma^{3}\right)
$$

It should also be noted that the chiral combination $\sigma^{1}-i \sigma^{2}$ ensures (3.4). This is essential since $G^{2}$ is the source of the dilaton, and this is constant in the five dimensional solution.

The stress tensor for the 5 -form alone is

$$
T_{m n}^{(5)}=4 m^{2} \Omega^{-10} g_{m n}, \quad T_{a b}^{(5)}=-4 m^{2} \Omega^{-10} g_{a b}
$$


where in our conventions ( $c f$. (2.15) $) m, n$ represent flat indices along $A d S_{5}$, while $a, b$ are flat indices along the internal manifold.

The self-duality of $F$ implies that $F_{M_{1} \ldots M_{5}} F^{M_{1} \ldots M_{5}}=0$ so that the only contribution to the scalar curvature comes from the 3-index tensor,

$$
R=\frac{1}{24}|G|^{2}
$$

Substituting this back into the Einstein equations (3.1) along the $A d S_{5}$ directions determines $m$ (in (3.9)) and fixes the $A d S_{5}$ radius, $L$, in terms of $a=\sqrt{2} L_{0}$ :

$$
m=\frac{4}{9} \frac{2^{1 / 3}}{L_{0}}, \quad L=\frac{3}{22^{2 / 3}} L_{0}
$$

This generates the proper value of the cosmological constant for the $A d S_{5}$ (cf. (2.5)):

$$
\frac{\Lambda}{\Lambda_{0}}=\left(\frac{L}{L_{0}}\right)^{2}=\frac{42^{4 / 3}}{9}
$$

By examining the Einstein equations (3.1) along the internal space one can eliminate $a_{1}(\theta)$ in terms of $a_{2}(\theta)$. Substituting this into the Maxwell equations (3.3) yields one second order and two first order differential equations for $a_{2}(\theta)$, and these can be integrated. The result is

$$
a_{1}(\theta)=-\frac{22^{1 / 3}}{9} i L^{2} \cos \theta, \quad a_{2}(\theta)=-i \frac{\sin (2 \theta)}{3-\cos (2 \theta)} a_{1}(\theta) .
$$

Our result for the $B$-field is particularly simple when written in terms of frames:

$$
B=-\frac{i}{\sqrt{3}} e^{-2 i \phi}\left(e^{6}+i e^{9}\right) \wedge\left(e^{7}-i e^{8}\right) .
$$

With this $B$-field all the equations of motion are satisfied, and we have a solution that has the correct global symmetry and cosmological constant. It remains to verify that the solution is indeed supersymmetric. 


\section{Supersymmetry}

To find the unbroken supersymmetry we must solve the equations $\delta \psi_{\mu}=0 ; \delta \lambda=0$ in the IIB supergravity theory. These equations have the form (for vanishing dilaton and axion) 13:

$$
D_{M} \epsilon+\frac{i}{480} F_{P Q R S T} \gamma^{P Q R S T} \gamma_{M} \epsilon+\frac{1}{96} G_{P Q R}\left(\gamma_{M}^{P Q R}-9 \delta_{M}^{P} \gamma^{Q R}\right) \epsilon^{*}=0,
$$

and

$$
\gamma^{M N P} G_{M N P} \epsilon=0 .
$$

We adopt exactly the same conventions as [13, except that our frame indices in (2.15) run from $1, \ldots, 10$ as opposed to $0, \ldots, 9$.

We start by solving (4.2). From (3.14) it follows that:

$$
G=d B=\omega \wedge B
$$

where

$$
\omega=-\frac{3 i}{L \Omega}\left(e^{10}-i \sqrt{\frac{2}{3}} \frac{\sin (2 \theta)}{(3-\cos (2 \theta))} e^{6}\right) .
$$

One can check that when contracted into $\gamma$-matrices the differential form $\omega \wedge\left(e^{6}+i e^{9}\right)$ yields an invertible operator (at least for $\sin (2 \theta) \neq 0$ ). It follows that (4.2) is satisfied if and only if the supersymmetry generators satisfy:

$$
\left(\gamma^{7}-i \gamma^{8}\right) \epsilon=0
$$

This selects a 16 (real) parameter subspace of chiral spinors.

The $S U(2) \times U(1)$ invariance of the background implies that $\epsilon=\epsilon\left(x^{\mu}, r, \theta, \phi\right)$ does not depend on the $S U(2) \times U(1)$ directions. Moreover, the supersymmetry generators are a doublet of $S L(2, \mathbb{R})$, and this can be used to fix the $\phi$-dependence of $\epsilon$ just as we did in the Ansatz for $G$. Similarly, the superconformal invariance on the $A d S_{5}$ fixes the dependence on $x^{\mu}, r, \mu=1, \ldots, 4$. These facts can also be verified by direct computation using (4.1).

The solutions for $\epsilon$ are most easily determined by taking carefully chosen linear combinations of the equations (4.1), i.e.:

$$
\alpha_{M} \gamma^{M} \delta \psi_{M}+\alpha_{N} \gamma^{N} \delta \psi_{N}=0
$$


where there is no summation over repeated indices. One chooses the constants $\alpha_{M}$ and $\alpha_{N}$ so that the terms with the 5-index tensor, $F$, cancel. For the directions along the brane (4.6) reduces to

$$
\gamma^{m} \frac{\partial}{\partial x^{m}} \epsilon=\gamma^{n} \frac{\partial}{\partial x^{n}} \epsilon, \quad m, n=1, \ldots, 4,
$$

while with one parallel and one in the transverse $r$-direction (4.6) becomes

$$
2 L \gamma^{m} \frac{\partial}{\partial x^{m}} \epsilon=e^{r / L} \gamma^{5}\left(2 L \frac{\partial}{\partial r}-1\right) \epsilon \quad m=1, \ldots, 4
$$

The general solution to these equations is a linear combination of

$$
\begin{gathered}
\epsilon^{(+)}=e^{r /(2 L)} \xi^{(+)} \\
\epsilon^{(-)}=\left(e^{r /(2 L)} \frac{x^{\mu} \gamma_{\mu}}{L}+e^{-r /(2 L)} \gamma^{5}\right) \xi^{(-)}
\end{gathered}
$$

where $\xi^{( \pm)}=\xi^{( \pm)}(\theta, \phi)$ are $S U(2)$ invariant chiral spinors, $\gamma^{11} \xi^{( \pm)}=\mp \xi^{( \pm)}$, that depend on the internal coordinates only.

Using similar difference of variations along the brane and an $S U(2)$ direction, and simplifying the derivative terms using (4.9) or (4.10) and the independence on the $S U(2)$ coordinates, yields an algebraic constraint:

$$
\left(\sqrt{2} \cos \theta\left(\gamma^{5}-i \gamma^{10}\right)-\sqrt{3} \sin \theta\left(\gamma^{6}+i \gamma^{9}\right)\right) \xi^{( \pm)}=0
$$

The two obvious solutions to this constraint are given by constant spinors $\eta^{(+)}$and $\eta^{(-)}$ which satisfy

$$
\left(\gamma^{5}-i \gamma^{10}\right) \eta^{( \pm)}=0, \quad\left(\gamma^{6}+i \gamma^{9}\right) \eta^{( \pm)}=0, \quad\left(\gamma^{7}-i \gamma^{8}\right) \eta^{( \pm)}=0
$$

where, for completeness, we have included (4.5). It follows from this and the helicity of $\epsilon$ (i.e. $\left.\gamma^{11} \epsilon=-\epsilon\right)$ that:

$$
\gamma^{1} \gamma^{2} \gamma^{3} \gamma^{4} \eta^{( \pm)}= \pm i \eta^{( \pm)}
$$

The constraints (4.12) reduce the original 32 supersymmetries by a factor of 8 to four supersymmetries. Indeed, the spinors, $\eta^{( \pm)}$, have opposite chirality along the brane and each is thus a single complex Weyl spinor in four dimensions.

Associated to each solution, $\eta^{( \pm)}$, of the constraints 4.12 is a "conjugate spinor" that also solves (4.11):

$$
\tilde{\eta}^{( \pm)}=\left(\sqrt{2} \cos \theta \gamma^{5}-\sqrt{3} \sin \theta \gamma^{6}\right) \gamma^{7}\left(\eta^{( \pm)}\right)^{*}
$$


Note that because of the complex conjugation, $\tilde{\eta}^{( \pm)}$has opposite brane-helicity to $\eta^{( \pm)}$.

At this point we have the building blocks of the unbroken supersymmetry. One takes a linear combinations of $\eta^{( \pm)}$and $\tilde{\eta}^{( \pm)}$with coefficients that are arbitrary functions of $\theta$ and $\phi$ and substitutes them into the remaining supersymmetry variations. The result is a system of algebraic and first-order differential equations. The solution is

$\xi^{( \pm)}(\theta, \phi)=(3-\cos (2 \theta))^{-3 / 8}\left(e^{-2 i \phi} \sin \theta \eta^{( \pm)}-i\left(\sqrt{2} \cos \theta \gamma^{5}-\sqrt{3} \sin \theta \gamma^{6}\right) \gamma^{7}\left(\eta^{( \pm)}\right)^{*}\right)$

Remember that $\eta^{( \pm)}$are independent, complex Weyl spinors on the brane. Thus $\epsilon^{( \pm)}$ provide an $\mathcal{N}=2$ supersymmetry in the bulk, while $\epsilon^{(+)}$, which is independent of the brane coordinates (see (4.9)), represents the $\mathcal{N}=1$ supersymmetry on the brane.

Before concluding we wish to make two remarks about the form of these supersymmetry generators. First, when seeking supersymmetries in complicated backgrounds it is often easier to start by finding the brane supersymmetry generators (4.9). Having found these, the other bulk supersymmetry in $A d S_{5}$, (4.10), can be generated using the superconformal symmetry. For example, the conformal boost between the time and $r$ directions corresponds to the Killing vector:

$$
\begin{aligned}
K^{1} & =\frac{1}{2 L}\left(\sum_{a=1}^{4}\left(x^{a}\right)^{2}+L^{2} e^{-2 r / L}\right), \quad K^{5}=-x^{1}, \\
K^{i} & =\frac{1}{L} x^{1} x^{i}, \quad i=2,3,4 .
\end{aligned}
$$

The infinitessimal change of coordinates: $x^{\mu} \rightarrow x^{\mu}+\varepsilon K^{\mu}$ induces a boost in the vielbein frame. When this change of coordinates and local frame boost acts on the spinor $\epsilon^{(+)}$in (4.9) the result is the spinor $\epsilon^{(-)}$in $(4.10)$ with $\xi^{(-)}=\gamma^{1} \xi^{(+)}$.

The second issue has to do with dimensional reduction. When one reduces the tendimensional IIB action to five dimensions one must perform rescalings of almost all the fields by powers of the determinant of the metric on the internal dimensions. This is to make sure that the $\sqrt{g}$ factors for the internal dimensions are not present in the fivedimensional kinetic terms. This is the origin of the warp factors. In particular, if $\hat{\psi}_{u}$ is the five-dimensional spin-3/2 field then for the metric (2.6) one has $\hat{\psi}_{u}=\Omega^{-1 / 2} \psi_{u}$, and hence the supersymmetry generators must be similarly related $\hat{\epsilon}=\Omega^{-1 / 2} \epsilon$. This extra factor converts (4.15) into something a little more canonical. 


\section{Final comments}

As we indicated in the introduction, the solution we have exhibited here is primarily of significance because of the of its relationship to a non-trivial supersymmetric fixed point of large $\mathrm{N}, \mathcal{N}=4$ Yang-Mills. It is also significant in that it represents a highly nontrivial confirmation of consistent truncation: Our ten-dimensional solution has precisely the global symmetries, supersymmetries, cosmological constant, and indeed internal metric that were predicted from the five-dimensional gauged $\mathcal{N}=8$ supergravity [1]].

The expression for the complete ten-dimensional metric as a function of all the fivedimensional supergravity scalars was first given in [1], and indeed, shortly after writing [3], we computed the metric (2.6), and examined it in detail along the flow and at the fixed point. Similar results were recently re-derived in [11]. However, the highly nontrivial task in finding the solution presented here, and indeed in the general consistent truncation story, is to handle the tensor field backgrounds and supersymmetry generators beyond the linearized level, and then solve both the equations of motion and the equations $\delta \psi_{\mu}=0 ; \delta \lambda=0$. Our expression, (3.14), for the $B$-field is, in fact, rather simple when expressed in terms of frames. This feature almost certainly is a result of the high level of symmetry in our solution. However, the simplicity of our result gives hope that more general solutions might be attainable, and indeed the construction of the ten-dimensional version of the complete flow of [3] should be within reach. Work on this is continuing.

\section{Acknowledgements}

This work was supported in part by funds provided by the DOE under grant number DE-FG03-84ER-40168. 


\section{References}

[1] A. Khavaev, K. Pilch and N.P. Warner, New Vacua of Gauged $\mathcal{N}=8$ Supergravity in Five Dimensions, hep-th/9812038.

[2] O. Aharony, S. S. Gubser, J. Maldacena, H. Ooguri and Y. Oz, Large N Field Theories, String Theory and Gravity, hep-th/9905111.

[3] D. Z. Freedman, S. S. Gubser, K. Pilch, and N. P. Warner, Renormalization Group Flows from Holography-Supersymmetry and a c-Theorem, CERN-TH-99-86, hepth/9904017

[4] R. G. Leigh and M. J. Strassler, Exactly Marginal Operators and Duality in FourDimensional $N=1$ Supersymmetric Gauge Theory, Nucl. Phys. B447 (1995) 95; hep-th/9503121

[5] S. Gubser, private communication.

[6] A. Karch, D. Lust and A. Miemiec, New $N=1$ Superconformal Field Theories and their Supergravity Description, Phys. Lett. B454 (1999) 265, hep-th/9901041.

[7] P. van Nieuwenhuizen and N.P. Warner, New Compactifications of Ten-Dimensional and Eleven-Dimensional Supergravity on Manifolds which are not Direct Products Comm. Math. Phys. 99 (1985) 141.

[8] L.J. Romans, New Compactifications of Chiral $N=2, d=10$ Supergravity, Phys. Lett. $\underline{153 B}$ (1985) 392.

[9] L. F. Abbott and S. Deser, Stabilty of Gravity with a Cosmological Constant, Nucl. Phys. B195 (1982) 76;

G.W. Gibbons, C.M. Hull and N. P. Warner, The Stability of Gauged Supergravity Nucl. Phys. B218 (1983) 173;

W. Boucher, Positive Energy without Supersymmetry, Nucl. Phys. B242 (1984) 282;

L. Mezincescu and P.K. Townsend, Positive Energy and the Scalar Potential in Higher Dimensional (Super)Gravity Theories, Phys. Lett. 148B (1984) 55;

L. Mezincescu and P.K. Townsend, Stability at a Local Maximum in Higher Dimensional Anti-de Sitter Space and Applications to Supergravity, Ann. Phys. 160 (1985) 406.

[10] N.P. Warner, Renormalization Group Flows from Five-Dimensional Supergravity, talk presented at Strings '99, Potsdam, Germany, 19-25 Jul 1999; hep-th/9911240

[11] M. Cvetič, H. Lü and C.N. Pope, Geometry of the Embedding of Scalar Manifolds in $D=11$ and $D=10$, hep-th/0002099. 
[12] B. de Wit and H. Nicolai, On the Relation Between $d=4$ and $d=11$ Supergravity, Nucl. Phys. B243 (1984) 91;

B. de Wit, H. Nicolai and N.P. Warner, The Embedding of Gauged $N=8$ Supergravity into $d=11$ Supergravity, Nucl. Phys. B255 (1985) 29.

[13] J.H. Schwarz, Covariant Field Equations of Chiral $N=2, D=10$ Supergravity, CALT-68-1016, Nucl. Phys. $\underline{B 226}$ (1983) 269.

[14] B. de Wit, H. Nicolai, A New SO(7) Invariant Solution of $d=11$ Supergravity, Phys. Lett. 148B (1984) 60. 